\title{
Adsorption of polymers anchored to membranes
}

\author{
M. Breidenich, R.R. Netz, and R. Lipowsky ${ }^{a}$ \\ Max-Planck-Institut für Kolloid- und Grenzflächenforschung, 14424 Potsdam, Germany
}

Received 20 October 2000

\begin{abstract}
Polymers, which are attached to a membrane at one of their ends, exert an entropic pressure, which curves the membrane away from the polymers. It is shown that adsorption which arises from a short-ranged potential between the polymer and the membrane has a large influence on the curvature of the membrane, leading to a decrease of the entropically induced curvature. If one ignores the finite size of the anchor segment, the polymer-induced curvature does not change sign and vanishes in the limit of strong adsorption and a pure contact potential. If one includes the finite size of the anchor segment, the membrane bends towards the polymer for sufficiently strong adsorption.
\end{abstract}

PACS. 36.20.-r Macromolecules and polymer molecules - 05.40.-a Fluctuation phenomena, random processes, noise and Brownian motion - 82.70.-y Disperse systems

\section{Introduction}

Membranes, which are decorated by end-grafted polymers, provide simple model systems for biological membranes, such as the plasma membrane of the cell [1]. The backbone of the plasma membrane consists of a lipid bilayer which is typically about $4 \mathrm{~nm}$ thick. In order to protect the membrane from mechanical and chemical attack, the bilayer is coated by polysaccharides attached to proteins and lipids, which are embedded into the lipid bilayer.

In this paper, we investigate the influence of anchored polymers on the properties of the lipid bilayer [2]. It has been shown recently, that polymers, which are anchored to the membrane on one side, induce a curvature such that the membrane bends away from the polymers [3-6]. This effect is due to the entropic interaction between polymer and membrane, since both cannot penetrate each other.

In the case of anchored polymers, the membrane curvature is induced by changes in the configurational entropy of the polymer. This situation differs from the case of free polymers in solution, where the membrane curvature is determined by both the configurational and translational entropy of the polymers $[7,8]$. For this latter situation, contradictory results were obtained for the induced spontaneous curvature and for the rigidity of the membrane [9-13].

In the following, we extend our previous studies of the compound polymer/membrane system, in which the polymers are attached to the membrane. In addition to the entropic interaction between polymer and membrane, energetic interactions between the polymer segments and the membrane $[14,15]$ are now taken into account. To be spe-

\footnotetext{
${ }^{a}$ e-mail: lipowsky@mpikg-golm.mpg.de
}

cific, we will focus on short-ranged square well and contact potentials.

Our article is organized as follows. In Section 2, we first explain the basic molecular geometry of the polymermembrane system; the molecular details are found to be important since the polymer-induced curvature depends on several molecular length scales. In Section 3, we describe our systematic perturbative approach which allows us to calculate the membrane shape arising from the interactions with the anchored polymer. We then apply this method to contact potentials (Sect. 4) and square well potentials (Sect. 5). The analytic results obtained in this way are compared with the results of Monte Carlo simulations in Section 6. The final Section 7 contains a summary and a brief outlook.

\section{Molecular geometry and length scales}

The basic geometry of the polymer/membrane system studied in this article is shown in Figure 1. In this cartoon, an attempt has been made to visualize both the membrane and the polymer with molecular resolution. Inspection of this figure shows that the system is characterized by several molecular length scales. In polymer physics, one usually tries to focus on those properties which are independent of these small scales. We will show below, however, that the curvature induced by adsorbed polymers exhibits a subtle dependence on these molecular length scales. Therefore, we first present a short, qualitative discussion of these scales.

The flexible polymer chain anchored to the membrane is visualized as a chain of jointed segments which represent the skeleton of the polymer together with its side 


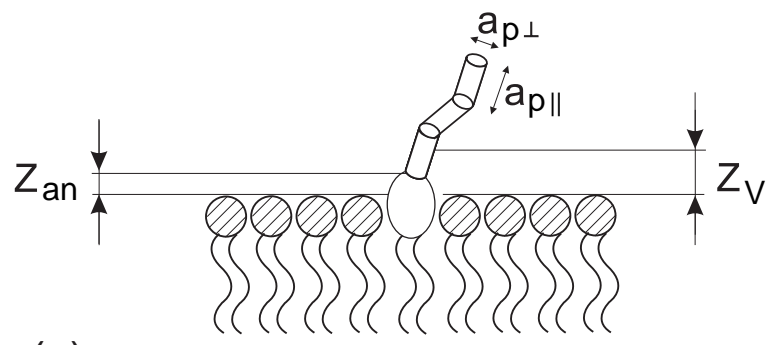

(a)

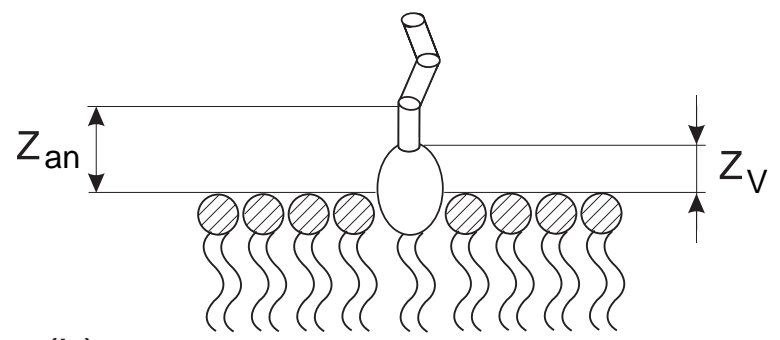

(b)

Fig. 1. The different molecular length scales of the polymer/membrane system. (a) A small head group of the anchor molecule and/or a flexible anchor/polymer bond can lead to $z_{\mathrm{an}}<z_{\mathrm{V}}$; and (b) a large head group of the anchor and/or a rigid anchor/polymer bond can lead to $z_{\text {an }}>z_{\mathrm{V}}$.

groups. The flexibility of the chain arises from the rotational isomerism of the jointed segments. The longitudinal size, $a_{\mathrm{p} \|}$, of each polymer segment is mainly determined by the length of the skeleton unit while the thickness, $a_{\mathrm{p} \perp}$, of such a segment will usually depend on the bulkiness of the sidegroups.

The flexible polymer chain is covalently bound to an anchor segment which must have a hydrophobic part in order to insert into the bilayer membrane. For a polymer anchored at one of its ends as studied here, the anchor segment typically consists of an amphiphilic molecule with a hydrophobic part inserted in the bilayer and a hydrophilic head group which provides the attachment site for the flexible polymer. This head group may have a size, $a_{\text {an }}$, which is comparable to or larger than the head groups of the lipid molecules as shown in Figure 1a. In addition, the covalent bond between the head group and the first segment of the polymer chain will usually not exhibit the same rotational isomerism as the jointed polymer segments. In particular, the chemical structure of this bond may not allow any bond rotation at all, as indicated in Figure 1b.

Now, consider the first polymer segment, which can exhibit several orientations with respect to the anchor segment, and its distance $z_{\text {an }}$ from the membrane surface. For the situation shown in Figure 1a, this distance is comparable to the size $a_{\text {an }}$ of the anchor head group; for the case in Figure $1 \mathrm{~b}, z_{\mathrm{an}} \simeq a_{\mathrm{an}}+a_{\mathrm{p} \|}$.

Finally, the system is characterized by the range $z_{\mathrm{V}}$ of the interaction potential between one polymer segment and the membrane surface. This interaction potential can exhibit (i) short-ranged contributions arising from direct contacts between polymer side groups and membrane surface and (ii) long-ranged contributions arising from van der Waals forces between the various molecular subgroups. In general, one will expect that $z_{\mathrm{V}}$ is of the order of $a_{\mathrm{p} \|}$.

In the following, we will use a continuum description for the polymer chain in which we basically ignore the molecular scales $a_{\mathrm{p} \|}$ and $a_{\mathrm{p} \perp}$. We will, however, incorporate (i) the potential range $z_{\mathrm{V}}$ and (ii) the anchoring distance $z_{\text {an }}$ into our theoretical description. Somewhat surprisingly, we find, both from analytical calculations and from computer simulations, that the sign of the polymer-induced curvature depends on the relative size of $z_{\mathrm{V}}$ and $z_{\text {an }}$. This behavior, which, at first sight, seems to be counter-intuitive, can be understood from the small free energy differences between the polymer-membrane conformations as discussed in Section 4.2 below.

\section{Perturbative approach}

The main property, which characterizes the shape of a membrane, is its curvature. The energy related to bending is governed by the bending rigidity $\kappa[16]$, which leads to the Hamiltonian

$$
\mathcal{H}_{\text {me }}\{l\}=\int \mathrm{d}^{2} x \frac{\kappa}{2}\left[\nabla^{2} l(\underline{x})\right]^{2}
$$

for the so called Monge parametrization of the membrane shape, where $l(\underline{x})$ is the distance between the neutral surface of the membrane and the two-dimensional reference plane with coordinates $\underline{x}=\left(x_{1}, x_{2}\right)$. The topology of the membrane is assumed to remain unchanged which implies that the Gaussian curvature stays constant and needs not be considered in our investigations. The Monge parametrization neglects configurations, in which the membrane forms overhangs. Such configurations are rather unlikely as long as the lateral size $L$ of the membrane is small compared to its persistence length [17]. If $L$ is increased towards the latter length scale, the bending rigidity is reduced by the shape fluctuations [18]. For most lipid bilayers, the value of the bending rigidity is found to be in a range of $\kappa \approx 10-20 k_{\mathrm{B}} T$, and the persistence length $\sim \exp \left(4 \pi \kappa / 3 k_{\mathrm{B}} T\right)[19]$ is rather large, which justifies the Monge parametrization.

As long as we do not confine the membrane by a potential $V_{\mathrm{me}}(z)$, the membrane can freely diffuse in the $z$ direction perpendicular to the plane of reference. In order to calculate expectation values such as the average height $\langle l(\underline{x})\rangle$, it is helpful to eliminate the contribution of free diffusion by subtracting the height of the anchor point $\langle l(\underline{0})\rangle$.

The polymer which is anchored on the membrane at one end is taken to be an ideal or Gaussian chain. This situation corresponds to polymers in $\theta$-solvents, where the excluded volume interactions are balanced by the van der Waals attractions between the monomers. The important quantity which characterizes the polymer configuration is its end-to-end distance $R_{\mathrm{p}}$, which is given by $a_{\mathrm{p}} \sqrt{N}$ for 
an unperturbed ideal chain, where $a_{\mathrm{p}}$ is the Kuhn length providing the average bond length, and $N$ is the number of monomers. Denoting the internal length of the polymer by $s$ in such a way that $s=0$ corresponds to the starting point and $s=1$ to the end-point of the polymer, we find for the partition function of the anchored polymer

$$
\begin{aligned}
& \mathcal{Z}_{\mathrm{p}}\{l\}= \int^{\prime} \mathcal{D}\{\mathbf{r}\} \delta\left[r_{1}(0)\right] \delta\left[r_{2}(0)\right] \delta\left[r_{3}(0)-l(\underline{0})\right] \\
& \times \exp \left[-\int_{0}^{1} \mathrm{~d} s\left(\frac{3}{2 R_{\mathrm{p}}^{2}}\left[\frac{\mathrm{d} \mathbf{r}(s)}{\mathrm{d} s}\right]^{2}+\frac{V(\mathbf{r}(s), l)}{T}\right)\right],
\end{aligned}
$$

where $T$ is the temperature in energy units and the potential $V(\mathbf{r})=N v(\mathbf{r})$ with the interaction potential per polymer segment $v$. The potential contains the energetic short ranged interaction between polymer and membrane. Of course, $V(\mathbf{r}(s), l)$ depends on the distance of the polymer bead located at $\mathbf{r}(s)$ to the membrane surface $l(\underline{x})$. The prime at the path integration indicates that $r_{3}(s) \geq$ $l\left(r_{1}(s), r_{2}(s)\right)+l_{\text {me }} / 2$ according to the impenetrability of polymer and membrane where $l_{\text {me }}$ denotes the membrane thickness. In the other directions the polymer is not confined and thus the integration extends from $-\infty$ to $+\infty$.

We normalize the polymer partition function $\mathcal{Z}_{\mathrm{p}}\{l\}$ by the half space partition function $\mathcal{Z}_{\mathrm{p}}\{l=0\}$ of a polymer anchored on a flat surface [20]. Expanding the partition function $\mathcal{Z}_{\mathrm{p}}$ to first order in $l$ and integrating out the polymer's degrees of freedom, we obtain the expression

$$
\begin{aligned}
\frac{\mathcal{Z}_{\mathrm{p}}\{l\}}{\mathcal{Z}_{\mathrm{p}}\{0\}} & \approx 1-\int_{0}^{1} \mathrm{~d} s \int_{-\infty}^{\infty} \mathrm{d}^{2} x P(s, \underline{x}) l(\underline{x}) \\
& \approx \exp \left(-\int_{0}^{1} \mathrm{~d} s \int_{-\infty}^{\infty} \mathrm{d}^{2} x P(s, \underline{x}) l(\underline{x})\right),
\end{aligned}
$$

which is, in fact, an expansion up to first order in the gradients $\partial l / \partial x_{1}$ and $\partial l / \partial x_{2}[5]$ (here and below, the symbol $\approx$ stands for asymptotically equal). The function $P$ is the pressure (measured in units of $T$ ) which the polymer exerts on the membrane and is given by

$$
\begin{aligned}
P(s, \underline{x})= & \frac{9}{2 \pi R_{\mathrm{p}}^{4}}\left(\frac{\mathrm{d}}{\mathrm{d} s}\langle z(s)\rangle_{\mathrm{hs}}\right)\left(\frac{3}{2} \frac{x^{2}}{R_{\mathrm{p}}^{2}} \frac{1}{s^{3}}-\frac{1}{s^{2}}\right) \\
& \times \exp \left(-\frac{3}{2 s} \frac{x^{2}}{R_{\mathrm{p}}^{2}}\right) .
\end{aligned}
$$

The average distance $\langle z(s)\rangle_{\mathrm{hs}}$ of the polymer segment $s$ from the reference plane now contains the information on the potential. Irrespective of the special form of $\langle z(s)\rangle_{\mathrm{hs}}$, the $x$-dependence of (4) is always such that the integrated pressure vanishes, since no external forces are applied to the system.

The total partition function of the compound system is

$$
\begin{aligned}
\mathcal{Z}_{\mathrm{c}}= & \int \mathcal{D}\{l\} \exp \left(-\mathcal{H}_{\mathrm{me}}\{l\} / T\right. \\
& \left.-\int_{0}^{1} \mathrm{~d} s \int_{-\infty}^{\infty} \mathrm{d}^{2} x P(s, \underline{x}) l(\underline{x})\right) .
\end{aligned}
$$

The mean shape profile of the membrane is obtained by insertion of the pressure term (4) into (5), which yields

$$
\begin{aligned}
\langle l(x)\rangle-\langle l(0)\rangle= & -\frac{T}{8 \pi \kappa} \int_{0}^{1} \mathrm{~d} s\left(\frac{\mathrm{d}}{\mathrm{d} s}\langle z(s)\rangle_{\mathrm{hs}}\right) \\
& \times\left(\gamma+\Gamma\left[0, \frac{3}{2 s} \frac{x^{2}}{R_{\mathrm{p}}^{2}}\right]+\ln \left[\frac{3}{2 s} \frac{x^{2}}{R_{\mathrm{p}}^{2}}\right]\right)
\end{aligned}
$$

where $\gamma$ is the Euler constant and $\Gamma$ is the incomplete Gamma-function [21,22].

The rather simple $x$-dependence of equation (6) allows us to calculate the mean curvature $M(\underline{x})=-1 / 2\left\langle\nabla^{2} l(\underline{x})\right\rangle$ induced by the polymer and the integrated (spontaneous) curvature $\mathcal{A} \bar{M}=\int \mathrm{d}^{2} x \quad M(\underline{x})$ where $\mathcal{A}$ denotes the surface area of the membrane. This leads to

$$
\mathcal{A} \bar{M}=\frac{T}{4 \kappa}\left(\langle z(1)\rangle_{\mathrm{hs}}-z_{\mathrm{an}}\right)
$$

which relates the membrane curvature to the average $z$ distance of the last polymer bead, i.e. the free end of the polymer in the half-space, and to the anchoring distance $z_{\text {an }}$.

The polymer partition function in the $z$-direction satisfies the Schrödinger-type equation

$$
\left[\partial_{s}-\frac{R_{\mathrm{p}}^{2}}{6} \partial_{z}^{2}+\frac{V(z)}{T}\right] \mathcal{Z}\left(z_{\mathrm{an}}, z \mid s\right)=0 \quad \text { for } 0 \leq s \leq 1
$$

So far we did not specify the explicit form of the potential. In the following we will consider two cases. First, we investigate a contact potential with vanishing range. In a further step we extend the calculation to the case of a square well potential with energy step $w$ and range $z_{\mathrm{V}}$. In particular we perform extensive Monte Carlo simulations in order to compare them with our analytic results.

\section{Contact potential}

The concept of a contact potential is an idealization. If one starts from a general potential given by

$$
V(z)=\left\{\begin{array}{lll}
\infty & \text { for } \quad z \leq 0 \\
V_{I}(z) & \text { for } \quad 0<z \leq z_{\mathrm{V}} \\
=0 & \text { for } & z_{\mathrm{V}}<z
\end{array}\right.
$$

one has to solve the Schrödinger-type equation in the corresponding regions and match the different solutions. As one can easily see, the solutions have to vanish at $z=0$. For $0<z<z_{V}$ it is not feasible to solve the equation in closed form for an arbitrary $z$-dependence of $V_{I}(z)$. However, it is possible to calculate the solution outside the potential range. The continuity and differentiability conditions lead to a length scale, the so-called extrapolation 
length $l_{\mathrm{ex}}$, which characterizes the solution inside the potential range without its explicit calculation [23]. At the same time, the extrapolation length, which now contains all the information on the specific form of $V_{I}(z)$, provides the boundary condition

$$
\left.\frac{\partial_{z} \mathcal{Z}\left(z_{\mathrm{an}}, z \mid s\right)}{\mathcal{Z}\left(z_{\mathrm{an}}, z \mid s\right)}\right|_{z=z_{\mathrm{V}}}=\frac{1}{l_{\mathrm{ex}}}
$$

for the outer solution with $z_{\text {an }}$ and $z>z_{\mathrm{V}}$. In the limit of vanishing potential range the $l_{\mathrm{ex}}$-dependent polymer partition function is given by [20]

$$
\begin{gathered}
\mathcal{Z}\left(z_{\mathrm{an}}, z \mid s\right)=\sqrt{\frac{3}{2 \pi s}} \frac{1}{R_{\mathrm{p}}}\left[\exp \left(-\frac{3}{2 s} \frac{\left(z-z_{\mathrm{an}}\right)^{2}}{R_{\mathrm{p}}^{2}}\right)\right. \\
\left.+\exp \left(-\frac{3}{2 s} \frac{\left(z+z_{\mathrm{an}}\right)^{2}}{R_{\mathrm{p}}^{2}}\right)\right]-\frac{1}{l_{\mathrm{ex}}} \exp \left(\frac{R_{\mathrm{p}}^{2}}{l_{\mathrm{ex}}^{2}} \frac{s}{6}+\frac{z+z_{\mathrm{an}}}{l_{\mathrm{ex}}}\right) \\
\times \operatorname{erfc}\left(\frac{R_{\mathrm{p}}}{l_{\mathrm{ex}}} \sqrt{\frac{s}{6}}+\sqrt{\frac{3}{2 s}} \frac{z+z_{\mathrm{an}}}{R_{\mathrm{p}}}\right)
\end{gathered}
$$

which allows us to calculate the expectation value of the polymer end point in the half space.

We will start with the situation of a polymer directly anchored to the membrane surface and later extend the investigations to a finite size of the anchor segment.

\subsection{Zero anchoring distance}

In the limit in which the anchoring distance $z_{\text {an }}$ is small compared to all other length scales, which corresponds to the case of direct surface anchoring, insertion of equation (11) into equation (7) leads to

$$
\mathcal{A} \bar{M} \approx \frac{T}{4 \kappa} l_{\mathrm{ex}}\left(\frac{\exp \left(-R_{\mathrm{p}}^{2} / 6 l_{\mathrm{ex}}^{2}\right)}{\operatorname{erfc}\left(R_{\mathrm{p}} / \sqrt{6} l_{\mathrm{ex}}\right)}-1\right)
$$

which no longer depends on $z_{\text {an }}$. The corresponding profile of $\mathcal{A} \bar{M}\left(R_{\mathrm{p}} / l_{\mathrm{ex}}\right)$ is displayed in Figure 2. The inverse extrapolation length $1 / l_{\text {ex }}$ provides a measure for the distance from the adsorption/desorption transition which occurs at $1 / l_{\mathrm{ex}}=0$. As one can already see in equation $(7)$, the spontaneous curvature is positive for all values of $1 / l_{\mathrm{ex}}$, since the expectation value of the polymer end point in the half-space cannot be negative. Thus, the membrane always bends away from the polymer.

In the limit of strong adsorption, the average shape profile of the membrane is flat. The expectation value of the polymer end-point is zero. In fact, all polymer beads are located on the membrane surface and consequently do not exert any pressure on it.

In the limit of strong desorption, we recover the result of a pure entropic interaction between polymer and membrane [5].

For $1 / l_{\mathrm{ex}}=0$, i.e., at the adsorption/desorption transition, it is possible to calculate the corresponding polymer

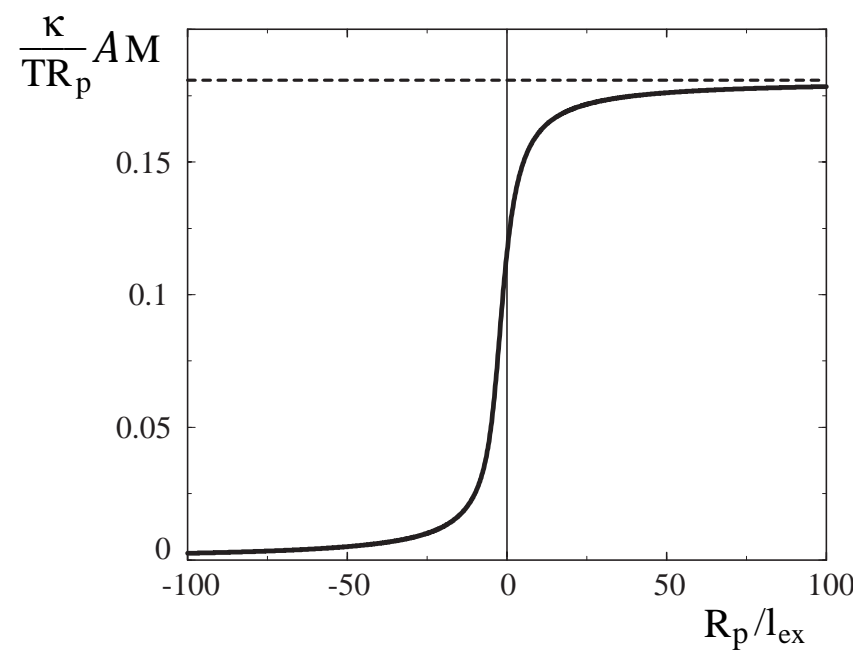

Fig. 2. The integrated curvature plotted versus the inverse extrapolation length $R_{\mathrm{p}} / l_{\mathrm{ex}}$ as obtained by the perturbative calculation. The dashed line denotes the limiting curvature for strong desorption.

pressure

$$
P(\underline{x})= \begin{cases}-\infty & x=0 \\ \frac{1}{3}\left(\frac{3}{2 \pi}\right)^{3 / 2} \frac{1}{x^{3}}\left(\frac{x^{3}}{R_{\mathrm{p}}^{3}}\left(3+\frac{R_{\mathrm{p}}^{2}}{x^{2}}\right) \exp \left(-\frac{3}{2} \frac{x^{2}}{R_{\mathrm{p}}^{2}}\right)\right. & \\ \left.+\sqrt{\frac{\pi}{6}} \operatorname{erfc}\left(\sqrt{\frac{3}{2}} \frac{x}{R_{\mathrm{p}}}\right)\right) & x>0\end{cases}
$$

and the resulting membrane shape profile

$$
\begin{aligned}
&\langle l(x)\rangle-\langle l(0)\rangle=- \frac{R_{\mathrm{p}}}{4 \pi} \frac{T}{\kappa}\left\{\frac{x}{R_{\mathrm{p}}} \operatorname{erfc}\left(\sqrt{\frac{3}{2}} \frac{x}{R_{\mathrm{p}}}\right)\right. \\
&+\frac{1}{\sqrt{6 \pi}} {\left[\gamma+2\left(1-\exp \left(-\frac{3}{2} \frac{x^{2}}{R_{\mathrm{p}}^{2}}\right)\right)\right.} \\
&\left.\left.+\mathrm{E}\left(1, \frac{3}{2} \frac{x^{2}}{R_{\mathrm{p}}^{2}}\right)+\ln \left(\frac{3}{2} \frac{x^{2}}{R_{\mathrm{p}}^{2}}\right)\right]\right\}
\end{aligned}
$$

where $E$ is the exponential integral function [22]. The shape profile is displayed in Figure 3 . In addition, we plotted corrections for small $1 / l_{\text {ex }}$ which were obtained numerically. The limiting behavior for the shape profiles shown above is always cone-like in the vicinity of the anchor and catenoid-like far away from it.

Thus, within the self-consistent scheme described here, the polymer-induced curvature has the same sign for all values of the inverse extrapolation length $1 / l_{\text {ex }}$. This differs from our previous calculation in which the shape of the membrane was constrained to be a spherical or conical segment: [14]. For these constrained shapes, one finds that the curvature changes sign at $l_{\mathrm{ex}}=0$ (if one ignores a possible curvature dependence of $\left.1 / l_{\mathrm{ex}}\right)$. Within the selfconsistent scheme, a similar curvature switch is now found as a function of the anchoring distance of the polymer as shown in the next subsection. 


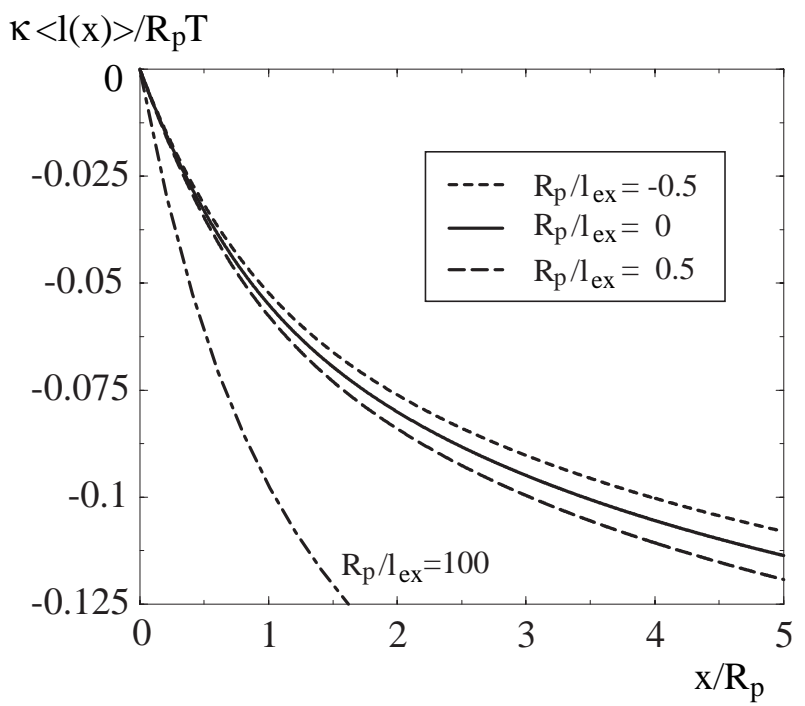

Fig. 3. The membrane shape profile close to the adsorption/desorption transition at $R_{\mathrm{p}} / l_{\mathrm{ex}}=0$. For comparison, the shape profile for strong desorption is also displayed.

\subsection{Nonzero anchoring distance}

In the experimental situation, the polymer is not necessarily anchored immediately on the membrane surface, but the anchor segment might protrude from the membrane such that the anchoring distance $z_{\text {an }}$ from the surface is nonzero. We will study the spontaneous curvature of the membrane induced by polymers with an initial distance $z_{\text {an }}$ of the starting point from the membrane.

The membrane spontaneous curvature is given by equation (7) in which $\langle z(1)\rangle_{\mathrm{hs}}$ depends parametrically on $z_{\text {an }}$. Using the partition function (11) for the averaging one finds

$$
\begin{aligned}
& \mathcal{A} \bar{M}\left(z_{\mathrm{an}}\right)=\frac{T}{4 \kappa}\left(z_{\mathrm{an}}+l_{\mathrm{ex}}\right)\left[-1+\left(\operatorname{erf}\left(\sqrt{\frac{3}{2}} \frac{z_{\mathrm{an}}}{R_{\mathrm{p}}}\right)\right.\right. \\
& \left.\left.\quad+\exp \left(\frac{R_{\mathrm{p}}^{2}}{6 l_{\mathrm{ex}}^{2}}+\frac{z_{\mathrm{an}}}{l_{\mathrm{ex}}}\right) \operatorname{erfc}\left(\frac{R_{\mathrm{p}} / l_{\mathrm{ex}}+3 z_{\mathrm{an}} / R_{\mathrm{p}}}{\sqrt{6}}\right)\right)^{-1}\right] .
\end{aligned}
$$

The spontaneous curvature as a function of the anchoring distance $z_{\text {an }}$ is shown in Figure 4 for different values of $R_{\mathrm{p}} / l_{\mathrm{ex}}$. The important result is the change in the sign of the curvature for nonzero $z_{\text {an }}$. For large $z_{\text {an }}$ the curvature approaches zero, since the polymer is not able to influence the membrane anymore. It is easy to show that the curvature sign changes at $z_{\text {an }}=-l_{\text {ex }}$. Thus, we only obtain a negative curvature where the membrane bends towards the polymer, if we are in the adsorption region with $R_{\mathrm{p}} / l_{\mathrm{ex}}<0$. For $R_{\mathrm{p}} / l_{\mathrm{ex}} \geq 0$ the curvature stays positive for all values of $z_{\mathrm{an}}$. In Figure 4 the limiting plot for $R_{\mathrm{p}} / l_{\mathrm{ex}}=0$ is given by the solid curve. For strong adsorption the curvature becomes strongly negative for non-vanishing values of $z_{\mathrm{an}}$.

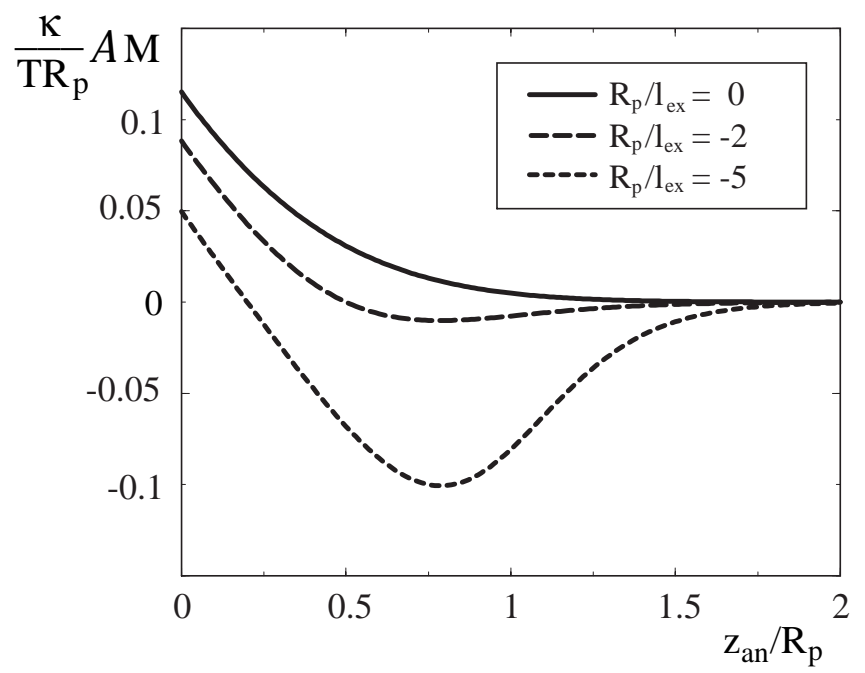

Fig. 4. The integrated (spontaneous) membrane curvature as a function of the anchoring distance $z_{\text {an }}$ for different values of the inverse extrapolation length $R_{\mathrm{p}} / l_{\mathrm{ex}}$. The curvature changes sign at $z_{\text {an }}=-l_{\text {ex }}$ in the adsorption regime. The limiting plot for $R_{\mathrm{p}} / l_{\mathrm{ex}}=0$ is shown in the solid curve.

Thus, an increase in the anchoring distance $z_{\text {an }}$ can change the sign of the polymer-induced curvature of the membrane. At first sight, this behavior, which is also found via Monte Carlo simulations, see Section 6 below, is somewhat surprising since a change in the anchoring distance corresponds to a rather local perturbation of the polymer conformations. It is important to note, however, that the corresponding free energy differences are relatively small, since they are of the order of $T^{2} / \kappa$, and, thus, can be affected by a few degrees of freedom.

Indeed, it follows from equation (3) that the free energy difference $\Delta F$ between the deformed and the planar membrane shape is given by $\Delta F=$ $T \int_{0}^{1} \mathrm{~d} s \int_{-\infty}^{\infty} \mathrm{d}^{2} x \quad P(s, \underline{x}) l(\underline{x})$. Dimensional analysis then implies the general scaling form

$$
\Delta F=\left(T^{2} / \kappa\right) \Phi\left(z_{\mathrm{an}} / R_{\mathrm{p}}, R_{\mathrm{p}} / \ell_{\mathrm{ex}}\right) .
$$

For the completely desorbed state with $1 / \ell_{\mathrm{ex}}=\infty$, one finds $\Phi(x, \infty)=(1 / 4 \pi) \ln (x)$ for a cone with a lateral membrane area corresponding to $\sim R_{\mathrm{p}}^{2}$ as previously calculated in reference [3].

So far, we have focussed on the behavior of the membrane segment which contains a single anchored polymer. Now, consider vesicles covered with $N_{\mathrm{p}}$ polymers. We consider polymers anchored on the membrane in the adsorption regime. If the polymer is anchored in a distance $z_{\mathrm{an}}=\left|l_{\mathrm{ex}}\right|+\delta_{z}$ with $\delta_{z}>0$ and both $\left|l_{\mathrm{ex}}\right|$ and $\delta_{z}$ are small compared to $R_{\mathrm{p}}$, it is guaranteed that the induced curvature is negative. The adsorption-induced curvature is given by

$$
\bar{M}^{(\mathrm{ad})}=-\frac{T}{4 \kappa} \delta_{z} B \Gamma_{\mathrm{p}}
$$

where $\Gamma_{\mathrm{p}}=N_{\mathrm{p}} / \mathcal{A}$ is the polymer coverage and $B$ denotes the absolute value of the square bracket in 
equation (15). Furthermore, the curvature induced by the repulsive polymer/polymer interaction yields a contribution which is quadratic in the coverage density,

$$
\bar{M}^{(\mathrm{pp})} \approx \frac{T}{4 \kappa} b_{2} R_{\mathrm{p}} \Gamma_{\mathrm{p}}^{2}
$$

where $b_{2}=4 \pi R_{\mathrm{p}}^{2}$ is the second virial coefficient, which characterizes the repulsive interaction in a low-density approximation. Since the latter contribution leads to the opposite curvature compared to $\bar{M}^{(\text {ad) }}$ the membrane curvature changes its sign as a function of the coverage density. The density at which the sign changes is given by

$$
\Gamma_{\mathrm{p}}^{*} \approx \frac{B}{b_{2}} \frac{\delta_{z}}{R_{\mathrm{p}}}
$$

For smaller densities the curvature is negative and thus the membrane bends towards the anchored polymers whereas for higher coverage densities the membrane bends away from the polymers. Consequently, the polymer coverage $\Gamma_{\mathrm{p}}$ provides another possible curvature switch.

\section{Square well potential with zero anchoring distance}

In order to generalize the previous investigation on contact potentials to more realistic potentials, and in order to compare the analytic results with Monte Carlo simulations, we will now investigate adsorption in square well potentials with potential depth $w$, measured in units of $T$, and range $z_{\mathrm{V}}$.

We start with the stationary Schrödinger-type equation which results from equation (8) by Laplace transformation:

$$
\left[t-\frac{R_{\mathrm{p}}^{2}}{6} \partial_{z}^{2}+V(z)\right] G\left(z_{\mathrm{an}}, z \mid t\right)=\delta\left(z_{\mathrm{an}}-z\right) .
$$

The Greens functions are given by

$$
\begin{aligned}
G_{\mathrm{I}}= & A_{1} \sin \left(\sqrt{6} q \frac{z_{\mathrm{an}}}{R_{\mathrm{p}}}\right)\left[\cos \left(\sqrt{6} q \frac{z}{R_{\mathrm{p}}}\right)+A_{2} \sin \left(\sqrt{6} q \frac{z}{R_{\mathrm{p}}}\right)\right] \\
& \text { for } \quad z_{\mathrm{an}} \leq z \leq z_{\mathrm{V}}, \\
G_{\mathrm{II}}= & B \sin \left(\sqrt{6} q \frac{z_{\mathrm{an}}}{R_{\mathrm{p}}}\right) \exp \left(-\sqrt{6} k \frac{z}{R_{\mathrm{p}}}\right) \text { for } z_{\mathrm{an}} \leq z_{\mathrm{V}} \leq z \\
G_{\mathrm{III}}= & C_{1}\left[\exp \left(\sqrt{6} k \frac{z_{\mathrm{an}}}{R_{\mathrm{p}}}\right)+C_{2} \exp \left(-\sqrt{6} k \frac{z_{\mathrm{an}}}{R_{\mathrm{p}}}\right)\right] \\
& \times \exp \left(-\sqrt{6} k \frac{z}{R_{\mathrm{p}}}\right) \quad \text { for } \quad z_{\mathrm{V}} \leq z_{\mathrm{an}} \leq z .
\end{aligned}
$$

Here and in the following, we use the wavenumber inside the potential well $q=\sqrt{w-t}$ and the wavenumber outside the well $k=\sqrt{t}$.

Let us furthermore focus on the limiting case where the polymer is directly anchored on the membrane, i.e. the anchoring distance $z_{\text {an }}=0$ in order to eliminate one parameter from the problem. As a consequence, we only need $G_{\mathrm{I}}$ and $G_{\mathrm{II}}$ in order to construct the complete Greens function as given by

$$
G\left(z_{\mathrm{an}}, z \mid t\right)=G_{\mathrm{I}} \theta\left(z_{\mathrm{V}}-z\right)+G_{\mathrm{II}} \theta\left(z-z_{\mathrm{V}}\right) .
$$

The remaining task is to calculate the coefficients. The correct treatment of the $\delta$-function on the right-hand-side of equation (20) leads to the prefactors $A_{1}$ and $C_{1}$,

$$
A_{1}=\frac{\sqrt{6}}{R_{\mathrm{p}}} \frac{1}{q} \quad \text { and } \quad C_{1}=\frac{\sqrt{6}}{R_{\mathrm{p}}} \frac{1}{2 k} .
$$

The other constants are determined by the continuity and differentiability conditions at $z=z_{\mathrm{V}}$, which lead to

$$
\begin{aligned}
A_{2} & =\frac{q-k \cot \left(\sqrt{6} q z_{\mathrm{V}} / R_{\mathrm{p}}\right)}{k+q \cot \left(\sqrt{6} q z_{\mathrm{V}} / R_{\mathrm{p}}\right)}, \\
B & =\frac{\sqrt{6}}{R_{\mathrm{p}}} \frac{\exp \left(\sqrt{6} k z_{\mathrm{V}} / R_{\mathrm{p}}\right)}{\sin \left(\sqrt{6} q z_{\mathrm{V}} / R_{\mathrm{p}}\right)\left(k+q \cot \left(\sqrt{6} q z_{\mathrm{V}} / R_{\mathrm{p}}\right)\right)}, \\
C_{2} & =\exp \left(2 \sqrt{6} k \frac{z_{\mathrm{V}}}{R_{\mathrm{p}}}\right) \frac{k-q \cot \left(\sqrt{6} q z_{\mathrm{V}} / R_{\mathrm{p}}\right)}{k+q \cot \left(\sqrt{6} q z_{\mathrm{V}} / R_{\mathrm{p}}\right)} .
\end{aligned}
$$

In the desorption case and at the adsorption/desorption transition, the solution of the Schrödinger-type equation leads to a continuous spectrum of energies. The polymer partition function is given by the inverse Laplace transformation

$$
\mathcal{Z}\left(z_{\mathrm{an}}, z \mid s\right)=\frac{1}{2 \pi \mathrm{i}} \int_{\eta-\mathrm{i} \infty}^{\eta+\mathrm{i} \infty} \mathrm{d} t \exp (s t) G\left(z_{\mathrm{an}}, z \mid t\right),
$$

where $\eta$ is a small positive number.

However, at the adsorption/desorption transition the first bound state occurs with energy $E_{1}=-t_{1}=0$. The discrete energy levels are given by the solution of the transcendental equation

$$
k=-q \cot \left(\sqrt{6} q \frac{z_{\mathrm{V}}}{R_{\mathrm{p}}}\right) .
$$

The partition function for the discrete spectrum of $N_{\mathrm{b}}$ bound states with energies $E_{n}=-t_{n}=-\left(w-q_{n}^{2}\right)$ is given by

$$
\mathcal{Z}^{(\mathrm{b})}\left(z_{\mathrm{an}}, z \mid s \equiv 1\right)=\sum_{n=1}^{N_{\mathrm{b}}} \exp \left(-E_{n}\right) G_{n}^{(\mathrm{b})}\left(z_{\mathrm{an}}, z \mid E_{n}\right)
$$

with $G_{n}^{(\mathrm{b})}$ composed in analogy to equation (22) and

$$
\begin{aligned}
G_{n, I}^{(\mathrm{b})}= & \frac{6}{R_{\mathrm{p}}} \frac{z_{\mathrm{an}}}{R_{\mathrm{p}}} q_{n} \frac{2 q_{n} \cot \left(\sqrt{6} q_{n} \frac{z_{\mathrm{V}}}{R_{\mathrm{p}}}\right)}{\sqrt{6} \frac{z_{\mathrm{V}}}{R_{\mathrm{p}}} q_{n} \cot \left(\sqrt{6} q_{n} \frac{z_{\mathrm{V}}}{R_{\mathrm{p}}}\right)-1} \sin \left(\sqrt{6} q_{n} \frac{z}{R_{\mathrm{p}}}\right) \\
& \text { for } \quad z_{\mathrm{an}} \leq z \leq z_{\mathrm{V}}, \\
G_{n, I I}^{(\mathrm{b})}= & \frac{6}{R_{\mathrm{p}}} \frac{z_{\mathrm{an}}}{R_{\mathrm{p}}} q_{n} \frac{2 q_{n} \cos \left(\sqrt{6} q_{n} \frac{z_{\mathrm{V}}}{R_{\mathrm{p}}}\right)}{\sqrt{6} \frac{z_{\mathrm{V}}}{R_{\mathrm{p}}} q_{n} \cot \left(\sqrt{6} q_{n} \frac{z_{\mathrm{V}}}{R_{\mathrm{p}}}\right)-1} \\
& \times \exp \left(\sqrt{6} q_{n} \frac{z-z_{\mathrm{V}}}{R_{\mathrm{p}}} \cot \left(\sqrt{6} q_{n} \frac{z_{\mathrm{V}}}{R_{\mathrm{p}}}\right)\right) \\
& \text { for } \quad z_{\mathrm{an}} \leq z_{\mathrm{V}} \leq z .
\end{aligned}
$$




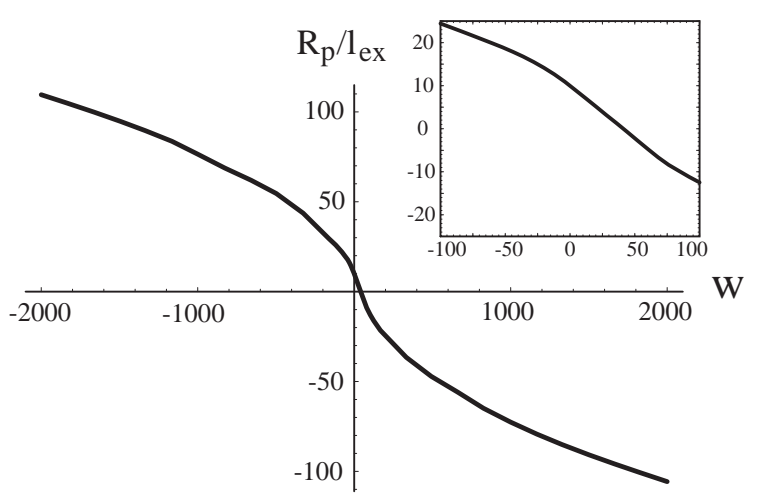

Fig. 5. The inverse extrapolation length $1 / l_{\text {ex }}$ as a function of the potential depth $w$ for fixed value of the potential range $z_{\mathrm{V}}=0.1 R_{\mathrm{p}}$, as used in the Monte Carlo simulations.

The value of the inverse extrapolation length is given by the logarithmic derivative of the solution inside the potential well evaluated at the first bound state which occurs, namely

$$
\frac{1}{l_{\mathrm{ex}}}=\frac{\sqrt{6} q_{1}}{R_{\mathrm{p}}} \cot \left(\sqrt{6} q_{1} \frac{z_{\mathrm{V}}}{R_{\mathrm{p}}}\right) .
$$

At the adsorption/desorption transition and in the desorption range, in which there are no bound states, the definition is extended to $q_{1}=\sqrt{w}$. In Figure 5 we plot the inverse extrapolation length as a function of the potential depth $w$ for fixed potential range $z_{\mathrm{V}}$.

Let us in the following discuss the three limiting situations of (i) strong desorption corresponding to a large positive inverse extrapolation length $1 / l_{\mathrm{ex}}$, (ii) the adsorption/desorption transition for $1 / l_{\mathrm{ex}}=0$ and (iii) strong adsorption for large negative $1 / l_{\mathrm{ex}}$. We will be interested in corrections to the result of the contact potential due to the finite potential range $z_{\mathrm{V}}$.

As mentioned, we restrict the investigation to the situation, in which the anchor segment of the polymer is small, Thus, we consider the polymer to be anchored directly on the membrane surface and $z_{\text {an }}=0$.

\subsection{Strong desorption}

As one can easily verify via equation (29), the limit of strong desorption, which corresponds to large positive $1 / l_{\mathrm{ex}}$, can be obtained in two ways:

For constant $w$, the inverse extrapolation length $1 / l_{\mathrm{ex}} \sim 1 / z_{\mathrm{V}}$ for small $z_{\mathrm{V}}$ and thus approaches the desorption case for large positive $1 / l_{\mathrm{ex}}$. If one inserts the expansions for small $z_{\mathrm{V}}$ into the solutions $G_{\mathrm{I}}$ and $G_{\mathrm{II}}$ of the Schrödinger-type equation, the expectation value $\langle z(1)\rangle_{\mathrm{hs}}$ for the polymer end point is found to behave as

$$
\begin{aligned}
\langle z(1)\rangle_{\mathrm{hs}} & =\frac{\int_{0}^{\infty} \mathrm{d} z z \mathcal{Z}(0, z \mid s=1)}{\int_{0}^{\infty} \mathrm{d} z \mathcal{Z}(0, z \mid s=1)} \\
& =\sqrt{\frac{\pi}{6}} R_{\mathrm{p}}+2 z_{\mathrm{V}} \frac{z_{\mathrm{V}}^{2}}{R_{\mathrm{p}}^{2}}|w|+\mathcal{O}\left(z_{\mathrm{V}}^{4}\right) .
\end{aligned}
$$

If this expansion is used in (7), one obtains the spontaneous curvature induced on the membrane as given by

$$
\mathcal{A} \bar{M} \approx \frac{1}{4} \frac{T}{\kappa} \sqrt{\frac{\pi}{6}} R_{\mathrm{p}}+\frac{1}{2} \frac{T}{\kappa} z_{\mathrm{V}} \frac{z_{\mathrm{V}}^{2}}{R_{\mathrm{p}}^{2}}|w|
$$

for small $z_{\mathrm{V}}$. As one expects, in the limit of vanishing $z_{\mathrm{V}}$ one recovers the result for strong desorption, i.e. the result where only the steric repulsion of polymer beads and the membrane surface are taken into account. This holds irrespective of the strength $w$ of the initial square well potential. If we now increase the range $z_{\mathrm{V}}$ without changing $w$, we also increase the mean $z$-distance of the last bead, which immediately leads to an increase in the induced spontaneous curvature.

There is another possibility of reaching the desorption limit, namely for fixed $z_{\mathrm{V}}$ and large negative $w$. Consequently, the polymer beads are repelled from the potential well. Inside the potential range the solution $G_{\mathrm{I}}$ vanishes for large negative $w$, i.e. there is no polymer bead inside the well except the anchor bead on the surface. The remaining contribution is due to $G_{\mathrm{II}}$. Inserting $q=\sqrt{w-t}=\mathrm{i} \sqrt{|w|+t}$ leads to the spontaneous curvature

$$
\mathcal{A} \bar{M} \approx \frac{1}{4} \frac{T}{\kappa}\left(\sqrt{\frac{\pi}{6}}-\frac{1}{\sqrt{6|w|}}\right) R_{\mathrm{p}}+\frac{1}{4} \frac{T}{\kappa} z_{\mathrm{V}}
$$

for large $|w|$. First, we note that we again recover the result for strong desorption from the leading term of this expansion.

Furthermore, equation (32) holds for all values of $z_{\mathrm{V}}$ in the limit of large $|w|$. For larger values of $z_{\mathrm{V}}$, this implies that the Gaussian polymer which is fixed by the anchor bead on the membrane surface, is strongly over-stretched in the first bond between the anchor and the following bead. This leads to the intuitive picture in which the end point of the polymer is shifted by the value of $z_{\mathrm{V}}$ in the $z$ direction. However, for large $z_{\mathrm{V}}$ this result is not physical anymore, since it arises from an artifact of the Gaussian model. The spring between anchor and first bead can be extended to infinity, which is not true for real polymers. The Gaussian model breaks down in this limit and is consequently not applicable anymore.

On the other hand, we expect the predictions of equation (32) to be correct for small $z_{\mathrm{V}}$. We will use this relation in order to compare our Monte Carlo data in the region of large positive $R_{\mathrm{p}} / l_{\mathrm{ex}}$ and fixed $z_{\mathrm{V}}=0.1 R_{\mathrm{p}}$.

\subsection{The behavior at the adsorption/desorption transition}

Here, we focus on the adsorption/desorption transition at $1 / l_{\mathrm{ex}}=0$. The adsorption/desorption transition, where the first bound state (with energy $t=0$ ) occurs, is given by the first zero of the righthandside of equation (29). The solution is

$$
\sqrt{6 w} \frac{z_{\mathrm{V}}}{R_{\mathrm{p}}}=\frac{\pi}{2}
$$


The advantage of this restriction to $1 / l_{\mathrm{ex}}=0$ is due to the possibility of varying $z_{\mathrm{V}}$ and $w$ according to equation (33) in such a way that $1 / l_{\text {ex }}$ stays constant. Inserting (33) into $q$ yields

$$
q=\sqrt{\frac{\pi^{2}}{24} \frac{R_{\mathrm{p}}^{2}}{z_{\mathrm{V}}^{2}}-t}=\frac{\pi}{2 \sqrt{6}} \frac{R_{\mathrm{p}}}{z_{\mathrm{V}}}-\frac{\sqrt{6}}{\pi} t \frac{z_{\mathrm{V}}}{R_{\mathrm{p}}}+\mathcal{O}\left(z_{\mathrm{V}}^{3}\right) .
$$

In order to calculate the effect on the curvature we again need $\langle z(1)\rangle_{\mathrm{hs}}$, i.e. the expectation value for the $z$-position of the polymer end point. Inserting the solutions and expanding in $z_{\mathrm{V}}$ leads to

$$
\langle z(1)\rangle_{\mathrm{hs}}=\sqrt{\frac{2}{3 \pi}} R_{\mathrm{p}}+\frac{(\pi+4)(\pi-2)}{2 \pi^{2}} z_{\mathrm{V}}+\mathcal{O}\left(z_{\mathrm{V}}^{2}\right) .
$$

As we expect, the first term of the expansion reproduces the result for vanishing $z_{\mathrm{V}}$ at $1 / l_{\mathrm{ex}}=0$. Since the curvature is given by equation (7) and the prefactor of the $z_{\mathrm{V}}$-term has the same sign as the term of order 1 , we conclude that the membrane curvature is increased further, if one increases $z_{\mathrm{V}}$.

There is another limiting case which is of interest to study, namely the limiting behavior of the curvature and membrane shape profile for large $z_{\mathrm{V}}$. It is clear by intuition that in this case we have to obtain the limit of strong desorption again, since the polymer is not affected by a potential gradient, i.e. by the corresponding force, anymore.

The only Greens function which survives the limiting process (if we neglect the asymptotic corrections in $z_{\mathrm{V}}$ ) is $G_{I}$. In fact, the insertion of the limiting $q=\mathrm{i} \sqrt{t}$ leads, as expected, to the half space propagator and, in consequence, to the same spontaneous curvature as for strong desorption.

\subsection{The limit of strong adsorption}

The limit of strong adsorption, corresponding to large negative $1 / l_{\text {ex }}$ is not governed by the continuum of scattering states but by the bound states of the Schrödinger-type equation (8).

Again, the main task is to calculate the average $z$-distance of the polymer endpoint in the half-space. Using the definition of equation (27) one finds

$$
\langle z(1)\rangle_{\mathrm{hs}}=\frac{\sum_{n=1}^{N_{\mathrm{b}}} \exp \left(-E_{n}\right) \int_{0}^{\infty} \mathrm{d} z z G_{n}^{(\mathrm{b})}\left(0, z \mid E_{n}\right)}{\sum_{n=1}^{N_{\mathrm{b}}} \exp \left(-E_{n}\right) \int_{0}^{\infty} \mathrm{d} z G_{n}^{(\mathrm{b})}\left(0, z \mid E_{n}\right)} .
$$

Since the bound states are given by the transcendental equation (26) it is not possible to calculate $\langle z(1)\rangle_{\mathrm{hs}}$ analytically. However, one limiting case is accessible, namely the important limit of small $z_{\mathrm{V}} \ll R_{\mathrm{p}}$. If we approximate the polymer as an infinitely long chain, which is justified for $z_{\mathrm{V}} \ll R_{\mathrm{p}}$ it is correct to apply the ground state approximation, i.e. it is justified to take into account only the lowest bound state with lowest energy $E_{1}$. This gives

$$
\langle z(1)\rangle_{\mathrm{hs}}=\frac{\int_{0}^{\infty} \mathrm{d} z z G_{1}^{(\mathrm{b})}\left(0, z \mid E_{1}\right)}{\int_{0}^{\infty} \mathrm{d} z G_{1}^{(\mathrm{b})}\left(0, z \mid E_{1}\right)} .
$$

For large negative $1 / l_{\mathrm{ex}}$ one finds $q_{1} \approx \pi R_{\mathrm{p}} / \sqrt{6} z_{\mathrm{V}}$. The limiting ground state solution, which remains, is

$$
G_{n, I}^{(\mathrm{b})}=2 \pi \frac{1}{z_{\mathrm{V}}} \frac{z_{\mathrm{an}}}{z_{\mathrm{V}}} \sin \left(\pi \frac{z}{z_{\mathrm{V}}}\right) .
$$

Inserting and performing the integration finally yields in the limit of strong adsorption the important result

$$
\langle z(1)\rangle_{\mathrm{hs}}=\frac{z_{\mathrm{V}}}{2} \quad \text { and } \quad \mathcal{A} \bar{M}=\frac{1}{8} \frac{T}{\kappa} z_{\mathrm{V}}
$$

Note that this result is correct also from an intuitive point of view. The limit of strong adsorption in a square well potential of range $z_{\mathrm{V}}$ in the $z$-direction is equivalent to the situation of confining a polymer between two parallel plates in a distance $z_{\mathrm{V}}$. We expect the average position in the $z$-direction to be $z_{\mathrm{V}} / 2$.

However, if $z_{\mathrm{V}}$ is increased we have to take into account higher energy levels, at least the ground state and the neighboring level. The reason for this is the assumption of the infinite polymer length in ground state approximation. Irrespective of the size of $z_{\mathrm{V}}$ the expectation value always stays at $\langle z(1)\rangle_{\mathrm{hs}}=z_{\mathrm{V}} / 2$. An additional length scale which includes the finite end-to-end distance $R_{\mathrm{p}}$ does not occur. Only if we take into account the second energy level, the additional length scale occurs and respects the finite $R_{\mathrm{p}}$. This always leads to a lowering of $\langle z(1)\rangle_{\mathrm{hs}}$ as we expect. In particular for large $z_{\mathrm{V}}$ we expect the bound state contribution to lead to an expectation value which is identical with the result for strong desorption and thus governed only by $R_{\mathrm{p}}$ and not by $z_{\mathrm{V}}$ anymore. This is shown in the following subsection.

\subsection{Bound states for large potential range $z_{V}$}

Bound states in the limit of strong adsorption are given by the condition $q_{n}=n \pi R_{\mathrm{p}} / \sqrt{6} z_{\mathrm{V}}$ with integer $n$. The remaining solution in the limit of large $z_{\mathrm{V}}$ is $G_{I}$. Inserting $q_{n}$ one ends up with

$$
G_{n, I}^{(\mathrm{b})}=2 n \pi \frac{1}{z_{\mathrm{V}}} \frac{z_{\mathrm{an}}}{z_{\mathrm{V}}} \sin \left(n \pi \frac{z}{z_{\mathrm{V}}}\right) .
$$

Dividing numerator and denominator of equation (36) by $\exp \left(-E_{1}\right)$ yields the numerator

$$
\int_{0}^{z_{\mathrm{V}}} \mathrm{d} z z G_{1, I}^{(\mathrm{b})}+\sum_{n=2}^{N_{\mathrm{b}}} \exp \left(E_{1}-E_{n}\right) \int_{0}^{z_{\mathrm{V}}} \mathrm{d} z z G_{n, I}^{(\mathrm{b})} .
$$

The denominator follows in analogy. Insertion of the energy differences and integration lead to the average distance

$$
\langle z(1)\rangle_{\mathrm{hs}}=\frac{\sum_{n=1}^{\infty}(-1)^{n-1} \exp \left(-n^{2} x\right)}{\sum_{n=1}^{\infty} \exp \left(-n^{2} x\right)-\sum_{n=1}^{\infty}(-1)^{n} \exp \left(-n^{2} x\right)} z_{\mathrm{V}} .
$$




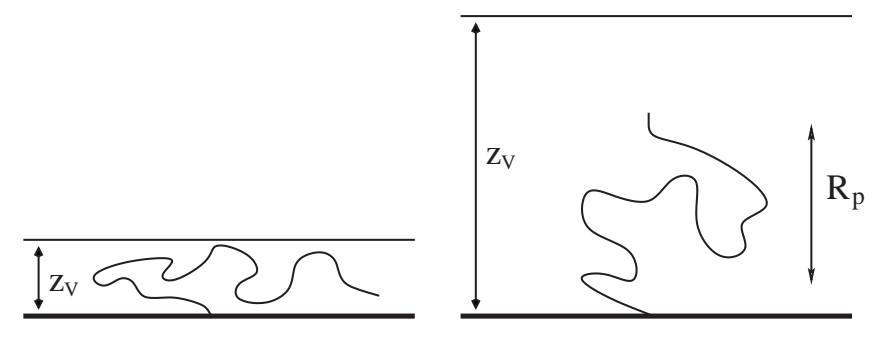

Fig. 6. Adsorption behavior in the limit of strong adsorption for $z_{\mathrm{V}} / R_{\mathrm{p}} \ll 1$ (left) and for $z_{\mathrm{V}} / R_{\mathrm{p}} \gg 1$ (right). The polymer on in the small slot is strongly deformed, whereas the polymer in the large slot is unperturbed.

It is possible to evaluate the sums analytically, which finally yields

$$
\langle z(1)\rangle_{\mathrm{hs}}=\sqrt{\frac{\pi}{6}} R_{\mathrm{p}} \quad \text { and } \quad \mathcal{A} \bar{M}=\frac{T}{4 \kappa} \sqrt{\frac{\pi}{6}} R_{\mathrm{p}} .
$$

The result recovers the limit of strong desorption. This is expected, since the polymer is not affected by the potential gradient anymore, if the potential step located at $z_{\mathrm{V}}$ is at a much larger distance from the surface than the polymer beads. Therefore we obtain the same polymer and membrane configuration as in the case of no adsorbing potential, see Figure 6 .

\section{Comparison with Monte Carlo simulation}

We will now compare the analytic results with Monte Carlo simulations. In the simulation, both the membrane and the polymer are discretized. The membrane can move continuously in the perpendicular direction above a two dimensional lattice $x^{(i, j)}$ with lattice constant $a_{\mathrm{m}}$. The configuration of the membrane is determined by its height $l^{(i, j)}$ above the lattice point $x^{(i, j)}$. We use periodic boundary conditions in the lateral directions.

The discretized Laplace operator which is used in the membrane Hamiltonian is given by

$$
\nabla_{d}^{2} l^{(i, j)}=l^{(i+1, j)}+l^{(i-1, j)}+l^{(i, j+1)}+l^{(i, j-1)}-4 l^{(i, j)} .
$$

The curvature energy difference for the membrane segment $(i, j)$ yields

$$
\begin{array}{r}
\Delta \mathcal{H}_{\mathrm{me}}=\kappa\left\{( \tilde { l } ^ { ( i , j ) } - l ^ { ( i , j ) } ) \left(l^{(i+2, j)}+l^{(i-2, j)}+l^{(i, j+2)}\right.\right. \\
+l^{(i, j-2)}+2\left[l^{(i+1, j+1)}+l^{(i-1, j+1)}+l^{(i+1, j-1)}\right. \\
\left.+l^{(i-1, j-1)}\right]-8\left[l^{(i+1, j)}+l^{(i-1, j)}+l^{(i, j+1)}\right. \\
\left.\left.\left.+l^{(i, j-1)}\right]+10\left[\tilde{l}^{(i, j)}+l^{(i, j)}\right]\right)\right\},
\end{array}
$$

where we take into account twelve next neighbors. We use a single-site-move algorithm, in which we compare the energies of two configurations $\left\{\tilde{l}_{N}\right\}$ and $\left\{l_{N}\right\}$, which differ in the randomly chosen value of $l$ at position $(i, j)$. In addition, one has to consider the impenetrability of polymer beads and membrane segment and the change in the polymer/membrane-interaction potential due to the move.

The decision whether or not to accept the new configuration follows the Metropolis-algorithm which ensures detailed balance.

The anchored polymer is described by the bead-spring model, in which the neighboring point like beads are coupled by a harmonic spring. This model corresponds to an ideal chain. The Kuhn length $a_{\mathrm{p}}$ provides the average bond length. One Monte Carlo step of the compound system corresponds to a single move of each membrane segment and each polymer bead. Averages calculated in the Monte Carlo simulations take into account $10^{7}$ Monte Carlo steps.

Due to the periodic boundary conditions we simulate a membrane covered by a regular lattice of anchored polymers in which the anchoring distance of the polymers is given by the lateral size of the membrane lattice. This size is chosen about eight times larger than the polymer endto-end distance $R_{\mathrm{p}}$, which corresponds to the dilute coverage (mushroom) regime, i.e. the polymers do not penetrate each other.

The square well potential, which acts on each bond, is included by measuring the distance in $z$-direction of a polymer bead and the membrane segment which is located beneath the bead. In this case, each bead represents the corresponding polymer bond, on which the potential acts. In order to approach the continuum limit, it is therefore necessary to choose the discretization of the polymer, i.e. the bond length, smaller than the potential range $z_{V}$.

The interesting quantity which we are going to measure is the induced spontaneous curvature of the membrane. The integrated curvature of all membrane segments vanishes, because of the periodic boundary conditions. Therefore, we choose an area of integration corresponding to a circle of radius $R_{\mathrm{p}}$, which is basically the area over which the polymer interacts with the membrane. The area contains the 13 inner membrane segments in the vicinity of the anchor. Results vary slightly if one increases the integration area, but do not change the diagram qualitatively, see the inset of Figure 7. The major contribution to the curvature arises from the cone-like bend of the anchor segment. It is therefore justified to choose the area small in order to avoid boundary effects. The integrated curvature as a function of the inverse extrapolation length is shown in Figure 7. The solid curve displays the analytic solution for the curvature in case of a contact potential. Since we do not use a contact potential in the simulation, but a square well of range $z_{\mathrm{V}}=0.1 R_{\mathrm{p}}$, the results of the contact potential will be shifted according to our analytic calculations. The shifts are displayed in the diagram for the limiting cases of strong adsorption and desorption, respectively, and for the adsorption/desorption transition. For strong adsorption, the curvature does not vanish, since the polymer can still move in a volume of height $z_{\mathrm{V}}$ above the membrane. Thus, the polymer still exerts a pressure on the membrane, leading to a small but positive curvature, analytically given by $z_{\mathrm{V}} T / 8 \kappa$ in the limit of large 


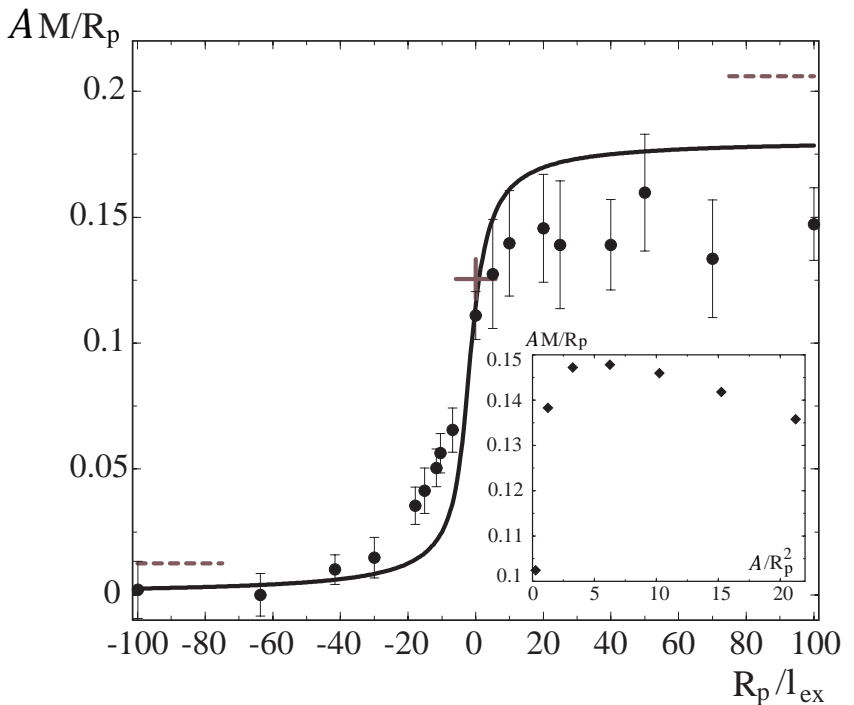

Fig. 7. The integrated curvature $\mathcal{A} \bar{M}$ versus the inverse extrapolation length $1 / l_{\mathrm{ex}}$ as obtained by the perturbative approach for a contact potential and by Monte Carlo simulations. The dashed lines in the limits of strong desorption and adsorption and the cross at the adsorption/desorption transition are the shifts due to the square well potential of range $z_{\mathrm{V}}=0.1 R_{\mathrm{p}}$, which is the potential used in the simulation. The Monte Carlo data is obtained for $\kappa / T=1$. The membrane discretization is $a_{\mathrm{m}} / R_{\mathrm{p}}=0.5$ and the polymer discretization $a_{\mathrm{p}} / R_{\mathrm{p}}=0.0625$. Averages take into account $10^{7} \mathrm{MC}$ steps. In the inset, the dependence of the integrated membrane curvature on the size of the integration area (approximately circular) is displayed for $R_{\mathrm{p}} / l_{\mathrm{ex}}=100$. Note, that due to periodic boundary conditions the curvature vanishes, if one integrates over the whole square lattice. Therefore, the curvature in the inset decreases again for larger integration area.

negative $1 / l_{\mathrm{ex}}$. For the values of $z_{\mathrm{V}}=0.1 R_{\mathrm{p}}$ this leads to a shift of $\mathcal{A} \overline{\Delta M}=0.0125 R_{\mathrm{p}} T / \kappa$, which we denoted by the dashed line in the left part of the diagram.

At the adsorption/desorption transition we have calculated the shift to higher curvatures if $z_{\mathrm{V}}>0$, which is given by $\mathcal{A} \overline{\Delta M} \approx 0.04 R_{\mathrm{p}} T / \kappa$, denoted by the cross in the diagram at $R_{\mathrm{p}} / l_{\mathrm{ex}}=0$.

For strong desorption the shift is given by $\mathcal{A} \overline{\Delta M}=$ $0.025 R_{\mathrm{p}} T / \kappa$ and denoted by the dashed line on the right side of the diagram.

There is a general trend that the simulation data lie below the analytic predictions. In addition to the already mentioned effect due to the choice of the integration area, there are two important reasons for the difference:

(i) Discretization effects: Both membrane and polymer discretization influence the $\mathrm{MC}$ results. As mentioned, the Kuhn length of the polymer $a_{\mathrm{p}}$ is always chosen smaller than the range of the potential $z_{\mathrm{V}}=0.1 R_{\mathrm{p}}$, such that $a_{\mathrm{p}}=0.0625 R_{\mathrm{p}}$, corresponding to 257 polymer beads.

The membrane discretization is determined by the lattice parameter $a_{\mathrm{m}}=0.5 R_{\mathrm{p}}$, corresponding to $289 \mathrm{mem}$ brane segments. For finer discretization of both we come closer to the analytic prediction.

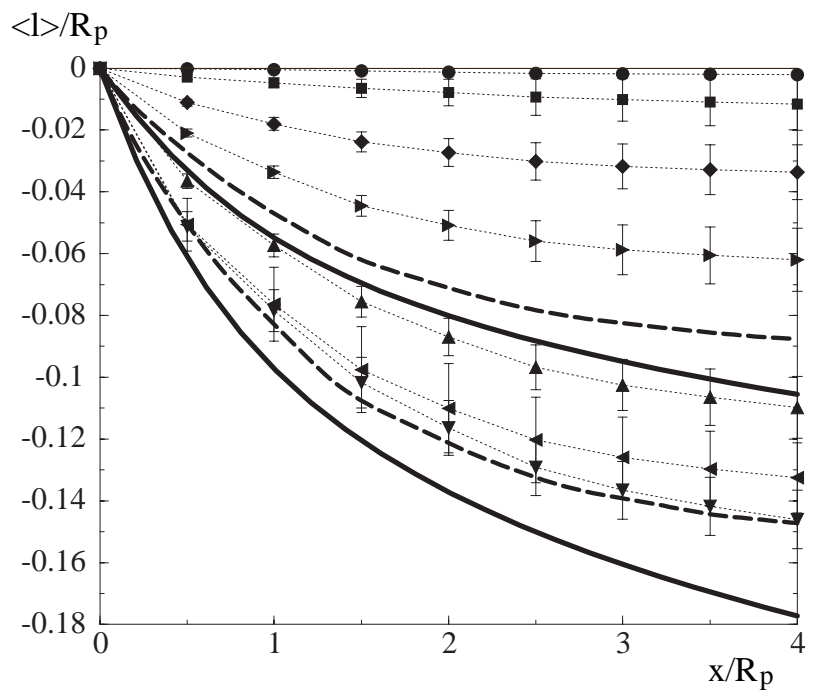

Fig. 8. Membrane shape profiles as obtained by MC simulations, using the same parameters as in Figure 7 for different values of $R_{\mathrm{p}} / l_{\mathrm{ex}}$ : (i) $R_{\mathrm{p}} / l_{\mathrm{ex}}=100$ (triangle down), (ii) $R_{\mathrm{p}} / l_{\mathrm{ex}}=25$ (triangle left), (iii) $R_{\mathrm{p}} / l_{\mathrm{ex}}=0$ (triangle up), (iv) $R_{\mathrm{p}} / l_{\mathrm{ex}}=-6.8$ (triangle right), (v) $R_{\mathrm{p}} / l_{\mathrm{ex}}=-17.8$ (diamond), (vi) $R_{\mathrm{p}} / l_{\mathrm{ex}}=-41.6$ (square) and (vii) $R_{\mathrm{p}} / l_{\mathrm{ex}}=-100$ (circle). The thin dashed lines in (i)-(vii) serve as a guide to the eye in order to distinguish the $\mathrm{MC}$ data sets for different values of $R_{\mathrm{p}} / l_{\mathrm{ex}}$. In addition, we display the analytically obtained profiles for a membrane with free boundaries (bold solid curves) and for a membrane with periodic boundaries, using the same lateral membrane size as in the simulations (bold dashed curves). The lower pair of bold curves shows the shape profile in the limit of strong desorption, using the same value of $R_{\mathrm{p}} / l_{\text {ex }}$ as in (i). The upper pair shows the shape profile at the adsorption/desorption transition, using the same value of $R_{\mathrm{p}} / l_{\mathrm{ex}}$ as in (iii).

(ii) Boundary effects: Since we use periodic boundary conditions the membrane shape profile differs from the free boundary case, which we use in the analytic calculation. This is especially important, since we did not confine the membrane by a harmonic potential, which leads to a finite parallel correlation length. If the parallel correlation length is small compared to the lateral membrane size one expects boundary effects to be small. For zero potential, on the other hand, the membrane is a self-similar object with diverging parallel correlation length and, thus, is always influenced by the system boundaries.

In Figure 8 we plot the membrane shape profile for different values of $R_{\mathrm{p}} / l_{\mathrm{ex}}$ as obtained by the $\mathrm{MC}$ simulation. In addition, the analytic predictions for strong desorption and for the adsorption/desorption transition are also included (i) for free membrane boundaries (bold solid curves) and (ii) for periodic boundaries, taking into account 30 Fourier modes (bold dashed lines). Close to the boundaries, the bold dashed profiles have vanishing slope. The slope difference in the anchor point is due to the finite number of Fourier modes which were taken into account. Comparing the simulation data with the analytic predictions at the adsorption/desorption transition and in the limit of strong desorption, which were both obtained 


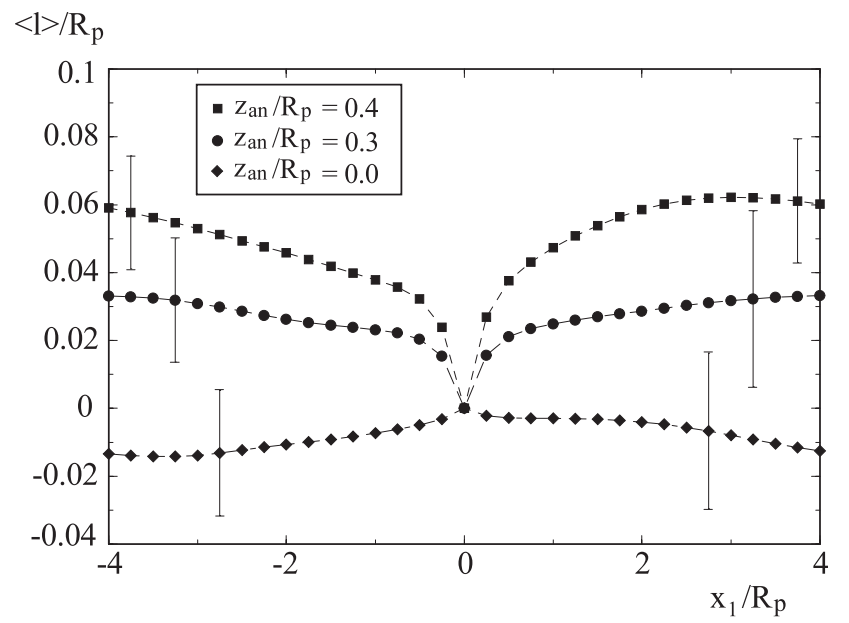

Fig. 9. Cross-sections of the membrane shape for different anchoring distances $z_{\mathrm{an}}$ of the polymer as obtained by Monte Carlo simulations. The shape profiles $\langle l\rangle$ and the lateral coordinate $x_{1}$ are measured in units of the end-to-end distance $R_{\mathrm{p}}$ of the polymer; the data are obtained by averaging over $6 \times 10^{6}$ Monte Carlo steps. The range of the square well potential is $z_{\mathrm{V}}=0.1 R_{\mathrm{p}}$, the strength is $R_{\mathrm{p}} / l_{\mathrm{ex}}=-30$, and $\kappa / T=1$. The discretization of the membrane in these simulations is of the same size as the polymer discretization: $a_{\mathrm{m}} / R_{\mathrm{p}}=a_{\mathrm{p}} / R_{\mathrm{p}}=0.25$.

for the case of a contact potential, the simulation curves slightly differ from the theoretical predictions, which corresponds to the observation in Figure 7.

Finally, in Figure 9, we present MC data for the dependence of the membrane shape on the reduced anchoring distance $z_{\mathrm{an}} / R_{\mathrm{p}}$ of the polymer. The range of the square well potential is the same as in the previous simulations. The strength of the potential is fixed to $R_{\mathrm{p}} / l_{\mathrm{ex}}=-30$. Membrane and polymer discretizations are chosen to be equal, $a_{\mathrm{m}} / R_{\mathrm{p}}=a_{\mathrm{p}} / R_{\mathrm{p}}=0.25$. In the figure, we display cross-sections of the averaged membrane profiles parallel to the $x_{1}$-direction. The profiles are obtained by averaging over $6 \times 10^{6}$ Monte Carlo steps. For zero anchoring distance $z_{\text {an }}=0$, the membrane bends away from the polymer as in the previous simulations. However, for the two nonzero values $z_{\text {an }}>0$ displayed in Figure 9, the membrane bends in the opposite direction, i.e., towards the polymer. Thus, the membrane curvature changes sign as a function of the anchoring distance $z_{\text {an }}$

\section{Conclusion and outlook}

We have shown that the spontaneous curvature which is induced by an anchored polymer is strongly influenced by the attractive interaction between polymer and membrane. In our perturbative approach, the spontaneous curvature is directly coupled to the average distance of the free polymer end point from the flat surface. The results on the induced membrane curvature sensitively depend on the distance $z_{\text {an }}$ of the anchor segment of the polymer from the membrane surface, see Figure 1. As long as the anchoring distance is small compared to all other molecular length scales, it is justified to neglect $z_{\text {an }}$ and to consider a polymer which is directly anchored on the membrane. In this limit, the membrane bends away from the polymer for all strengths of the interaction potential. For strong adsorption, the polymer does not induce any spontaneous curvature of the membrane.

For real systems, on the other hand, the anchoring distance $z_{\text {an }}$ may not be small compared to the other molecular length scales, see Figure 1. For $z_{\mathrm{an}}>0$, it is possible to change the membrane curvature towards the polymer in the adsorption regime. In this case, the polymer coverage density on the membrane can be used as a curvature switch, if one takes into account the sterically induced repulsive interaction between the polymers. One then finds that the membrane curvature changes its sign at the intermediate coverage as given by (19).

In order to study a more realistic system, and in order to compare the results with data from Monte Carlo simulations, we also studied adsorption in a square well potential of range $z_{\mathrm{V}}$ and depth $w$. For the limiting case $z_{\text {an }}=0$, in which the polymer is anchored directly on the membrane surface, the square well potential quantitatively, but not qualitatively, changes the results, which were obtained for vanishing $z_{\mathrm{V}}$ : The integrated membrane curvature is increased. Especially, in the limit of strong adsorption, the curvature does not vanish but has a small positive value, governed by the mean distance of the polymer end point above the surface $z_{\mathrm{V}} / 2$. These results are in good agreement with the MC simulations. These simulations also confirm that the polymer-induced curvature of the membranes changes sign as one increases the anchoring distance of the polymer.

Our theoretical study shows that the membrane curvature induced by an anchored polymer which is attracted towards the membrane surface depends on the molecular architecture of the polymer anchor. Within our models, this dependence arises from the relative size of the anchoring distance $z_{\mathrm{an}}$ and the range $z_{\mathrm{V}}$ of the polymer $/ \mathrm{mem}$ brane interaction potential. We have focussed here on the simplest types of interaction potentials as provided by square well potentials and by contact potentials corresponding to the limit of zero $z_{\mathrm{V}}$. In more realistic models, one would like to include (i) the precise spatial dependence of the interaction potential arising, e.g., from van der Waals forces or electrostatic forces, and (ii) the dependence of these interaction potentials on the curvature of the membrane surface. It is possible that these additional features of the interaction potential will again affect the sign of the polymer-induced curvature.

\section{Glossary: List of symbols}

The symbols are ordered alphabetically. Symbols with subscripts are treated as combined words.

$\begin{array}{ll}a_{\text {an }} & \text { size of the anchor head group } \\ a_{\mathrm{p}} & \text { Kuhn length } \\ \mathcal{A} & \text { area }\end{array}$




\begin{tabular}{|c|c|}
\hline $\mathcal{A} \bar{M}$ & integrated membrane curvature \\
\hline$b_{2}$ & second virial coefficient \\
\hline$E_{n}$ & discrete energy levels \\
\hline$G\left(z_{0}, z \mid t\right)$ & polymer Greens function with energy $-t$ \\
\hline$\Gamma_{\mathrm{p}}$ & polymer coverage density on the membrane \\
\hline $\mathcal{H}_{\text {me }}$ & membrane Hamiltonian \\
\hline$k$ & $\begin{array}{l}\text { wavenumber of the Greens function outside } \\
\text { the potential range }\end{array}$ \\
\hline$\kappa$ & bending rigidity \\
\hline$l$ & height of the neutral membrane surface \\
\hline$L$ & lateral membrane size \\
\hline$l_{\mathrm{ex}}$ & extrapolation length \\
\hline$l_{\text {me }}$ & membrane thickness \\
\hline$M$ & mean curvature \\
\hline$N$ & monomer number of the polymer \\
\hline$N_{\mathrm{b}}$ & number of discrete energy levels \\
\hline$N_{\mathrm{p}}$ & number of polymers \\
\hline$P$ & $\begin{array}{l}\text { polymer induced pressure on membrane } \\
\text { surface }\end{array}$ \\
\hline$q$ & $\begin{array}{l}\text { wavenumber of the Greens function inside } \\
\text { the potential range }\end{array}$ \\
\hline $\mathbf{r}(s)$ & spatial position of monomer $s$ \\
\hline$R_{\mathrm{p}}$ & polymer end-to-end distance \\
\hline$s$ & internal (contour) length of the polymer \\
\hline$t$ & negative energy $-E$ \\
\hline$T$ & $\begin{array}{l}\text { absolute temperature (generally in energy } \\
\text { units) }\end{array}$ \\
\hline$V$ & $\begin{array}{l}\text { interaction potential between polymer and } \\
\text { membrane }\end{array}$ \\
\hline$v_{2}$ & harmonic potential parameter \\
\hline$w$ & depth of the square well potential \\
\hline$\underline{x}$ & lateral directions $x_{1}, x_{2}$ \\
\hline $\bar{z}$ & perpendicular direction \\
\hline$z_{\text {an }}$ & anchoring distance of the polymer \\
\hline $\mathcal{Z}_{\mathrm{c}}$ & $\begin{array}{l}\text { compound partition function of polymer } \\
\text { and membrane }\end{array}$ \\
\hline $\mathcal{Z}_{\mathrm{p}}$ & polymer partition function \\
\hline $\mathcal{Z}_{\mathrm{hs}}$ & half-space polymer partition function \\
\hline$z_{\mathrm{V}}$ & range of the square well potential \\
\hline
\end{tabular}

\section{References}

1. B. Alberts, D. Bray, A. Johnson, J. Lewis, M. Raff, K. Roberts, P. Walter, Essential Cell Biology (Garland Publishing, Inc., New York, 1998).

2. R. Lipowsky, E. Sackmann, Structure and Dynamics of Membranes (Elsevier, Amsterdam, 1995).

3. R. Lipowsky, Europhys. Lett. 30, 197 (1995).

4. C. Hiergeist, R. Lipowsky, J. Phys. II France 6, 1465 (1996).

5. M. Breidenich, R.R. Netz, R. Lipowsky, Europhys. Lett. 49, 431 (2000).

6. T. Bickel, C. Marques, C. Jeppesen, Phys. Rev. E 62, 1124 (2000).

7. E. Eisenriegler, A. Hanke, S. Dietrich, Phys. Rev. E 54, 1134 (1996).

8. E. Eisenriegler, J. Phys. Cond. Matt. 12, A227 (2000).

9. P.G. de Gennes, J. Phys. Chem. 94, 8407 (1990).

10. J.T. Brooks, C.M. Marques, M.E. Cates, Europhys. Lett. 14, 713 (1991).

11. R. Podgornik, Europhys. Lett. 21, 245 (1993).

12. T. Garel, M. Kardar, H. Orland, Europhys. Lett. 29, 303 (1995).

13. F. Clement, J.-F. Joanny, J. Phys. II France 7, 973 (1997).

14. C. Hiergeist, V.A. Indrani, R. Lipowsky, Europhys. Lett. 36, 491 (1996).

15. Y.W. Kim, W. Sung, Europhys. Lett. 47, 292 (1999).

16. W. Helfrich, Z. Naturforsch. 28c, 693 (1973).

17. P.G. de Gennes, C. Taupin, J. Phys. Chem. US 86, 2294 (1982).

18. W. Helfrich, J. Phys. France 46, 1263 (1985).

19. L. Peliti, S. Leibler, Phys. Rev. Lett. 54, 1690 (1985).

20. E. Eisenriegler, Polymers near Surfaces (World Scientific, Singapore, 1993).

21. M. Abramowitz, I.A. Stegun, Handbook of mathematical functions (Dover Publications, Inc., New York, 1972).

22. I.S. Gradshteyn, I.M. Ryzhik, Table of Integrals, Series, and Products (Academic Press, San Diego, 1994).

23. P.G. de Gennes, Rep. Prog. Phys. 32, 187 (1969). 